\title{
EXISTENCE AND STABILITY OF BUSINESS CYCLES
}

\author{
BY \\ AKITAKA DOHTANI \\ Faculty of Economics, Toyama University, Japan
}

\begin{abstract}
We provide several sufficient conditions for the existence and the stability of limit cycles in two-dimensional differential equation systems. We also apply our results to business cycle models in the Keynesian tradition.
\end{abstract}

1. Introduction. So far, there have been many important papers on cyclical fluctuations in the business cycle theories in the Keynesian tradition. For brief surveys, see Gabisch and Lorenz [5], Lorenz [12], and Owase [14, 15]. Many of the papers discuss the endogenous cyclical fluctuations in economic activities as inevitable events in a capitalist system, by making the fluctuations correspond to the stable limit cycles of idealized two-dimensional models.

As a mathematical tool for detecting a limit cycle, we have the well-known PoincareBendixson theorem. To use this theorem, we need to detect a compact set on the boundary of which the vector field of a model points inward. Chang and Smyth [3] have provided, for the type of system $\Gamma: d x / d t=f(x, y), d y / d t=g(x, y)$, a geometrical method for searching for such a compact set. In Sec. 2 , we provide a more generalized sufficient condition for the existence of externally stable outermost and internally stable innermost limit cycles for $\Gamma$ than that obtained by utilizing the method of Chang and Smyth.

However, our first result (therefore, the result of Chang and Smyth [3]) does not hold true for the type $\Lambda: d x / d t=f(x, y), d y / d t=g(x)$, because in our first result the conditions on $g(x, y)$ with respect to $y$, such as $\partial g / \partial y<0$, are essentially utilized. This type of system has often appeared in the business cycle models in the Keynesian tradition. For example, see Schinasi [17]. Compared to $\Gamma$ with the conditions on $g(x, y)$ with respect to $y$, it is slightly more difficult to guarantee a limit cycle of $\Lambda$. Special forms of $\Lambda$, specifically the generalized Liénard system: $d x / d t=y-f(x), d y / d t=-g(x)$, have been elaborately studied by many mathematicians. See, for example, Sansone and Conti [16], Ye Yanqian [18], and Cesari [2]. However, the systems for which such studies have been intended are somewhat over specified from the viewpoint of economic dynamics. In Sec. 3, we present, for the most general form of $\Lambda$, an analytical sufficient condition for the existence of externally stable outermost and internally stable innermost cycles.

Received August 13, 1993.

1991 Mathematics Subject Classification. Primary 34C. 
It is more difficult to establish two-sided stability (merely stability) of an emerging cycle. As a tool for doing this, we have the Hopf bifurcation theorem. However, the stability of the limit cycle obtained from this theorem depends on the second and third derivatives of the vector field of a model. In economics, we can know at most second derivatives of it. Therefore, the Hopf bifurcation theorem does not give us any economically meaningful information on the stability of a limit cycle.

Another tool for investigating stability of a cycle is the uniqueness-stability theorems, which show the coincidence of externally stable outermost and internally stable innermost limit cycles. As for the Kaldor model, the results in this direction were first obtained by Lorenz [11]. Lorenz conjectured that, without the symmetric nonlinearities of investment and savings functions, the possibility of the existence of more than one limit cycle cannot be excluded. But more recently, Galeotti and Gori [6] succeeded in excluding such symmetric nonlinearities by utilizing the result by Zhang Zhifen [20]. Their result is, however, crucially dependent on ad hoc nonlinearities of the aggregate excess supply (= savings - investment) function, i.e., the left-hand concavity and the right-hand convexity of this function with respect to the equilibrium income. In Sec. 4, to exclude such nonlinearities, we present, by combining our existence theorem and Huang Kecheng's uniqueness theorem [18, p. 156], another sufficient condition that guarantees a unique stable limit cycle for the generalized Liénard system.

In the final section, we consider the existence and the stability of a cycle for two business cycle models in the Keynesian tradition. We first apply our results to the several different versions of the Kaldor model [3] and present an analytical sufficient condition for guaranteeing, for any of the versions, outermost externally stable and innermost internally stable cycles. Next, we show, by means of our unique existence theorem, that in the Kaldor model such nonlinearities as were assumed by Galeotti and Gori [6] are not always necessary for the existence of a unique stable limit cycle. Finally, we also provide an analytical sufficient condition for the existence of outermost externally stable and innermost internally stable cycles for the Schinasi model [17]. We also provide, for a specified version of the Schinasi model, a sufficient condition for the existence of a unique stable cycle.

2. Existence of outermost and innermost cycles. I. In this section, we provide a general sufficient condition for the existence of the outermost and innermost limit cycles for the two-dimensional differential equation system $\Gamma: d x / d t=f(x, y)$, $d y / d t=g(x, y)$.

For $\Gamma$, suppose $f$ and $g$ are everywhere of class $C^{1}$ and $f(0,0)=g(0,0)=0$.

Theorem 1. Suppose that $(0,0)$ is the unique equilibrium point of $\Gamma$. Under the following assumptions, $\Gamma$ has an outermost limit cycle that is externally stable and an innermost limit cycle that is internally stable.

(Г.1) $g(x, 0) x>0$ for any $x \neq 0, f(0, y) y<0$ for any $y \neq 0$.

(Г.2) $\lim _{y \rightarrow \pm \infty}|f(0, y)|=+\infty, \lim _{x \rightarrow \pm \infty}|g(x, 0)|=+\infty$. 
(Г.3) There exist positive numbers $\delta$ and $\zeta$ such that

(Г.3.1) $\operatorname{Sup}_{x \geq \delta, y \in \mathbf{R}^{\prime}}\{f(x, y)-f(0, y)\}<0$, $\operatorname{Inf}_{x \leq-\delta, y \in \mathbf{R}^{\prime}}\{f(x, y)-f(0, y)\}>0$,

(Г.3.2) $\operatorname{Sup}_{y \geq \zeta, x \in \mathbf{R}^{\prime}}\{g(x, y)-g(x, 0)\}<0$, $\operatorname{Inf}_{y \leq-\zeta, x \in \mathbf{R}^{\prime}}\{g(x, y)-g(x, 0)\}>0$,

(Г.3.3) $\operatorname{Sup}_{|x| \leq \delta, y \in \mathbf{R}^{\prime}}|f(x, y)-f(0, y)|<+\infty$, $\operatorname{Sup}_{|y| \leq \zeta, x \in \mathbf{R}^{\prime}}|g(x, y)-g(x, 0)|<+\infty$

( $\Gamma .4)$ Any eigenvalue of the Jacobian matrix of $(f, g)$ evaluated at $(0,0)$ has a positive real part.

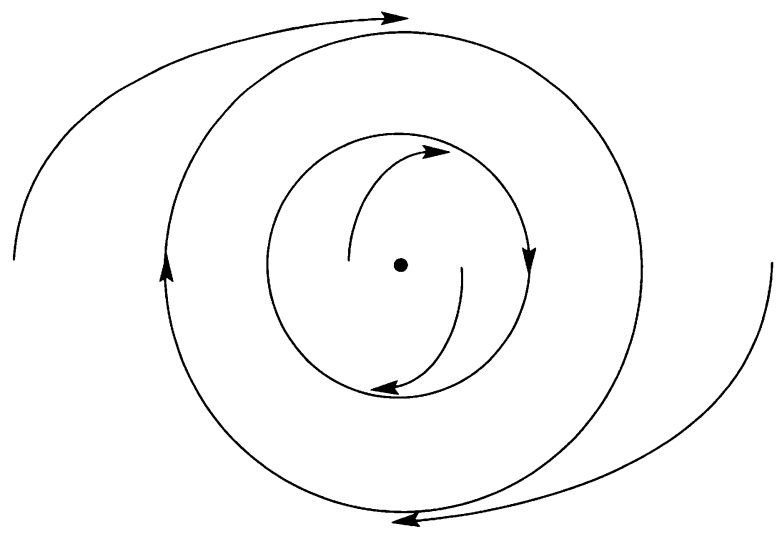

Fig. 1. Outermost and innermost limit cycles that are externally and internally stable, respectively. 


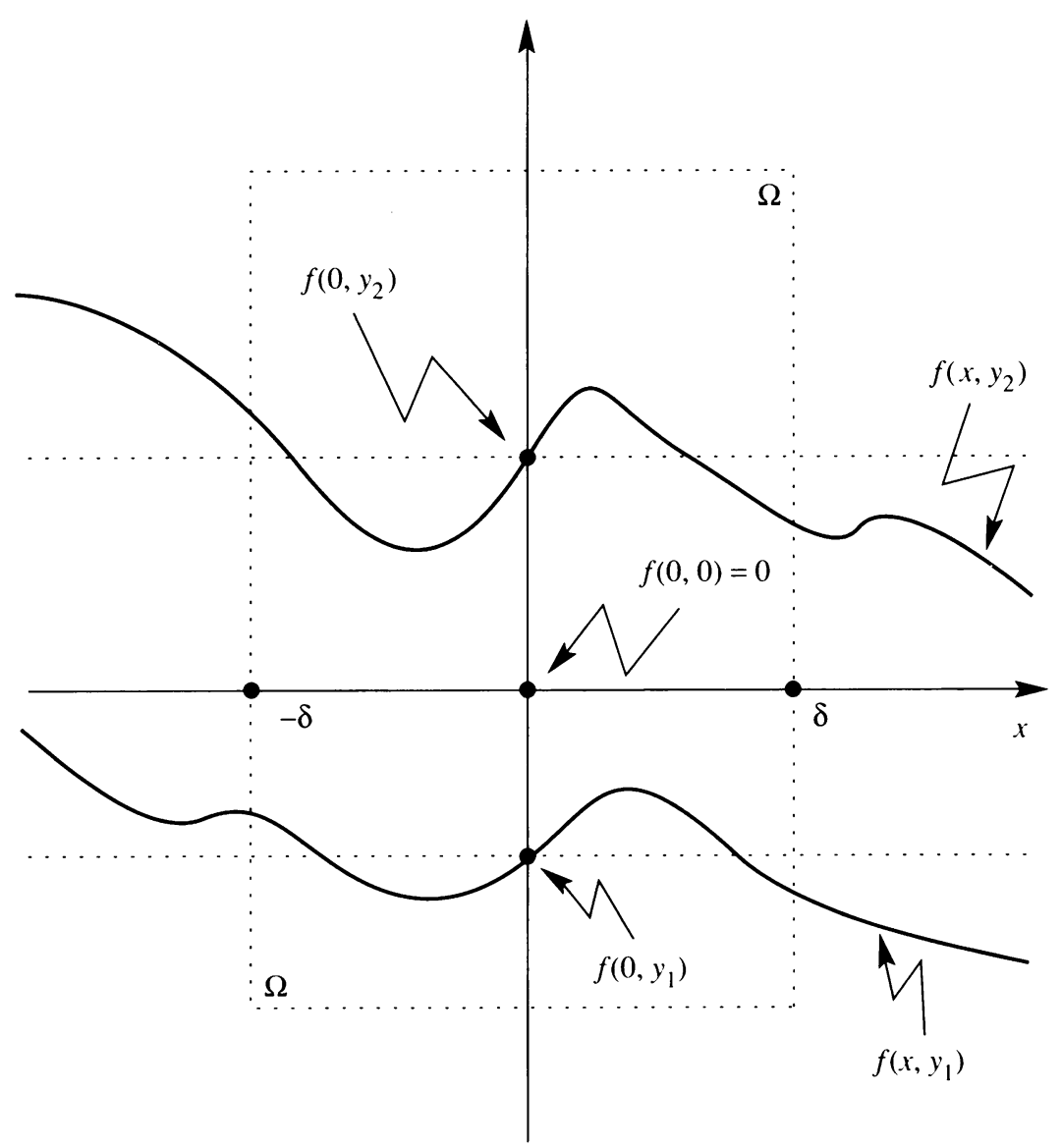

FIG. 2. Graphical illustration of Condition $\Gamma .3 . \quad \Omega$ is the set that contains $\{(x, f(x, y)):|x| \leq \delta\}$.

Proof. We see from $(\Gamma .4)$ that the positive limit set of any orbit with initial value $(x(0), y(0)) \neq(0,0)$ does not contain $(0,0)$.

Now, we prove the ultimate boundedness of solutions of $\Gamma$. From $(\Gamma .2)$ and ( $\Gamma .3)$, there are positive numbers $\eta(>\delta)$ and $\lambda(>\zeta)$ such that

$$
\begin{aligned}
|x| \geq \eta,|y| \leq \zeta \Rightarrow g(x, 0) & \{f(x, y)-f(0, y)\} \\
& +\operatorname{Sup}_{|y| \leq \zeta}|f(0, y)| \operatorname{Sup}_{|y| \leq \zeta, x \in \mathbf{R}^{\prime}}|g(x, y)-g(x, 0)|<0, \\
|x| \leq \delta,|y| \geq \lambda \Rightarrow-f(0, & y)\{g(x, y)-g(x, 0)\} \\
& +\operatorname{Sup}_{|x| \leq \delta}|g(x, 0)| \operatorname{Sup}_{|x| \leq \delta, y \in \mathbf{R}^{\prime}}|f(x, y)-f(0, y)|<0 .
\end{aligned}
$$

Fix $\eta$ and $\lambda$ that satisfy (1). Define the Liapunov function

Note that

$$
V(x, y)=\int_{0}^{x} g(p, 0) d p-\int_{0}^{y} f(0, q) d q .
$$

$$
\begin{aligned}
d V / d t & =g(x, 0) f(x, y)-f(0, y) g(x, y) \\
& =g(x, 0)\{f(x, y)-f(0, y)\}-f(0, y)\{g(x, y)-g(x, 0)\} .
\end{aligned}
$$




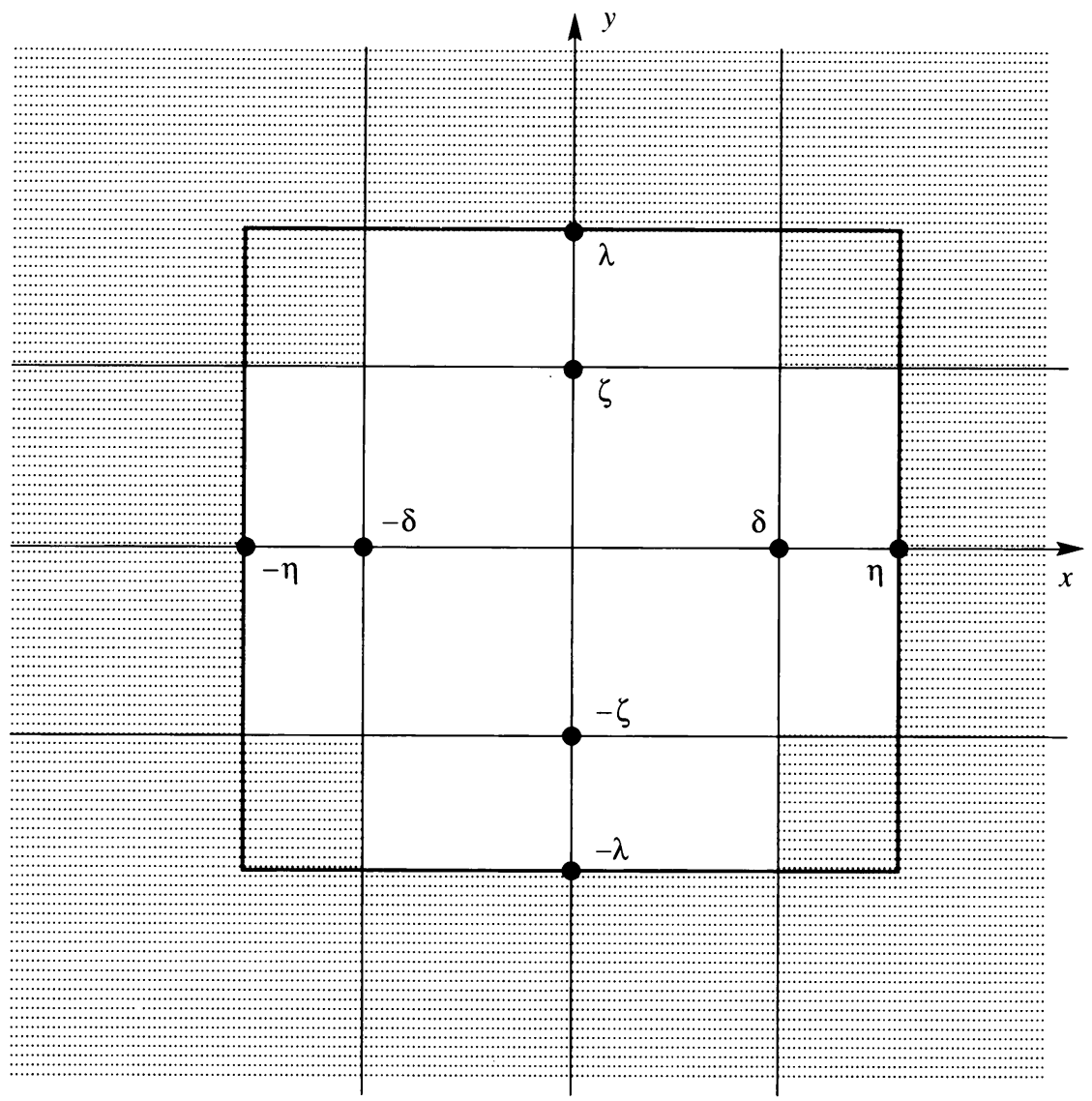

FIG. 3. Domain of negative time derivative (shaded).

Then, it follows directly from (1), (Г.3.1), and (Г.3.2) that

$$
(x, y) \in\left\{(x, y) \in \mathbf{R}^{2}:|x| \geq \eta\right\} \cup\left\{(x, y) \in \mathbf{R}^{2}:|y| \geq \lambda\right\} \Rightarrow d V / d t<0 .
$$

The ultimate boundedness of $\Gamma$ follows directly from (2) and Theorem 14.6 of Yoshizawa [19]. Then, the Poincare-Bendixson theorem completes the proof.

As shown later, $(\Gamma .3)$ and $(\Gamma .4)$ provide a mathematical generalization of the well-known Kaldor's condition.

The next corollary is useful for detecting limit cycles of the business cycle models in the Keynesian tradition.

Corollary 1. Suppose that $(0,0)$ is the unique equilibrium point of $\Gamma$. Under $(\Gamma .4)$ and the following conditions, $\Gamma$ has an outermost limit cycle that is externally stable and an innermost limit cycle that is internally stable.

(Г.5) $\operatorname{Inf}_{x \in \mathbf{R}^{\prime}} \frac{\partial g(x, 0)}{\partial x}>0, \operatorname{Sup}_{y \in \mathbf{R}^{\prime}} \frac{\partial f(0, y)}{\partial y}<0$.

(Г.6) There exist positive numbers $\delta$ and $\zeta$ such that

$$
\text { (Г.6.1) } \operatorname{Sup}_{|x| \geq \delta, y \in \mathbf{R}^{\prime}} \frac{\partial f(x, y)}{\partial x}<0, \operatorname{Sup}_{|y| \geq \zeta, x \in \mathbf{R}^{\prime}} \frac{\partial g(x, y)}{\partial y}<0,
$$


(Г.6.2) $\operatorname{Sup}_{|x| \leq \delta, y \in \mathbf{R}^{\prime}}\left|\frac{\partial f(x, y)}{\partial x}\right|<+\infty, \operatorname{Sup}_{|y| \leq \zeta, x \in \mathbf{R}^{1}}\left|\frac{\partial g(x, y)}{\partial y}\right|<+\infty$.

Proof. Note that

$f(x, y)-f(0, y)=\int_{0}^{x} \frac{\partial f(p, y)}{\partial x} d p, \quad g(x, y)-g(x, 0)=\int_{0}^{y} \frac{\partial g(x, q)}{\partial y} d q$.

The proof follows directly from Theorem 1 .

3. Existence of outermost and innermost cycles. II. Since $(\Gamma .3 .2)$ or $(\Gamma .6 .1)$ is assumed, Theorem 1 does not hold true for the type $\Lambda: d x / d t=f(x, y), d y / d t=$ $g(x)$, which has often appeared in the business cycle models in the Keynesian tradition. Similarly, the method of Chang and Smyth [3] does not hold true for $\Lambda$. Compared to $\Gamma$, it is slightly more difficult to obtain a sufficient condition for guaranteeing a limit cycle of $\Lambda$. Special forms of $\Lambda$ have been elaborately studied by many mathematicians. In this section, we provide an analytical condition for the existence of the outermost and innermost limit cycles for the most general form of $\Lambda$.

For $\Lambda$, suppose $f$ and $g$ are everywhere of class $C^{1}$ and $f(0,0)=g(0)=0$.

THEOREM 2. Under the following assumptions, $\Lambda$ has an outermost limit cycle that is externally stable and an innermost limit cycle that is internally stable.

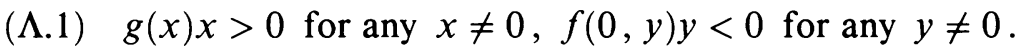

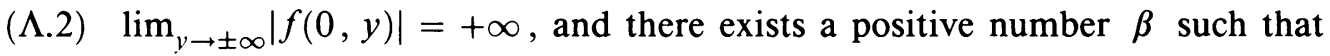
$\operatorname{Inf}_{|x| \geq \beta}|g(x)|>0$.

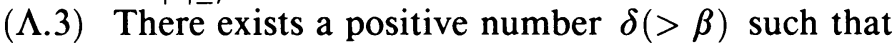
$(\Lambda .3 .1)$

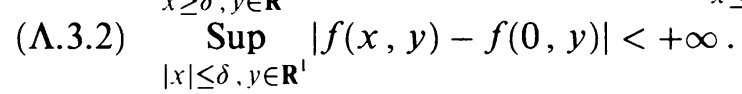$$
\text { Sup }\{f(x, y)-f(0, y)\}<0, \operatorname{Inf}_{x \leq-\delta, y \in \mathbf{R}^{\prime}}
$$
$\{f(x, y)-f(0, y)\}>0$

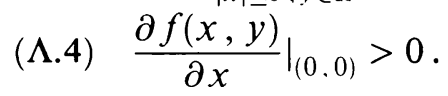

Proof. Note that by $(\Lambda .1),(0,0)$ is the unique equilibrium of $\Lambda$. Define

$$
u(x, y)=\int_{0}^{x} g(p) d p-\int_{0}^{y} f(0, q) d q .
$$

It follows from $(\Lambda .1)$ that $u>0$ for any $(x, y) \neq(0,0)$. We observe from $(\Lambda .4)$ that there exists a neighborhood of $(0,0)$, say $U(0,0)$, such that $\partial f(x, y) / \partial x>0$ for any $(x, y) \in U(0,0)$. Therefore, we have

$$
\frac{d u}{d t}=g(x)\{f(x, y)-f(0, y)\}>0 \quad \text { for any }(x, y) \in U(0,0) \cap\{x \neq 0\} .
$$

This implies that the positive limit set of any orbit with initial value $(x(0), y(0)) \neq$ $(0,0)$ does not contain $(0,0)$.

Next, we prove the ultimate boundedness of solutions of $\Lambda$. From ( $\Lambda .2)$ and

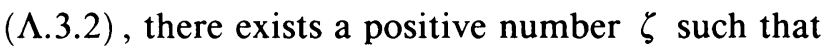

$$
\begin{aligned}
|x| \leq & \delta, y \geq \zeta \\
& \Rightarrow f(0, y)<-\left\{\operatorname{Sup}_{|x| \leq \delta, y \in \mathbf{R}^{\prime}}|f(x, y)-f(0, y)|\right\}\left\{\operatorname{Sup}_{|x| \leq \delta}|g(x)+1|\right\},
\end{aligned}
$$




$$
\begin{aligned}
|x| \leq & \delta, y \leq-\zeta \\
& \Rightarrow f(0, y)>\left\{\operatorname{Sup}_{|x| \leq \delta, y \in \mathbf{R}^{\prime}}|f(x, y)-f(0, y)|\right\}\left\{\operatorname{Sup}_{|x| \leq \delta}|g(x)-1|\right\} .
\end{aligned}
$$

Moreover, from $(\Lambda .1)$ and $(\Lambda .3 .1)$ there is a positive number $\theta$ such that

$$
x \leq-\delta \Rightarrow g(x)\{f(x, y)-f(0, y)-2 \delta / \theta\}<0 \text { for any } y \in \mathbf{R}^{1} .
$$

Putting $\eta=\operatorname{Max}\{\zeta, \theta\}$, define the Liapunov function

$$
V(x, y)= \begin{cases}u(x, y), & x \geq \delta, y \in \mathbf{R}^{1} \\ u(x, y)+x-\delta, & |x| \leq \delta, y \geq \eta \\ u(x, y)-2 \delta, & x \leq-\delta, y \geq \eta \\ u(x, y)-(2 \delta / \eta) y, & x \leq-\delta,|y| \leq \eta \\ u(x, y)+2 \delta, & x \leq-\delta, y \leq-\eta \\ u(x, y)-x+\delta, & |x| \leq \delta, y \leq-\eta\end{cases}
$$

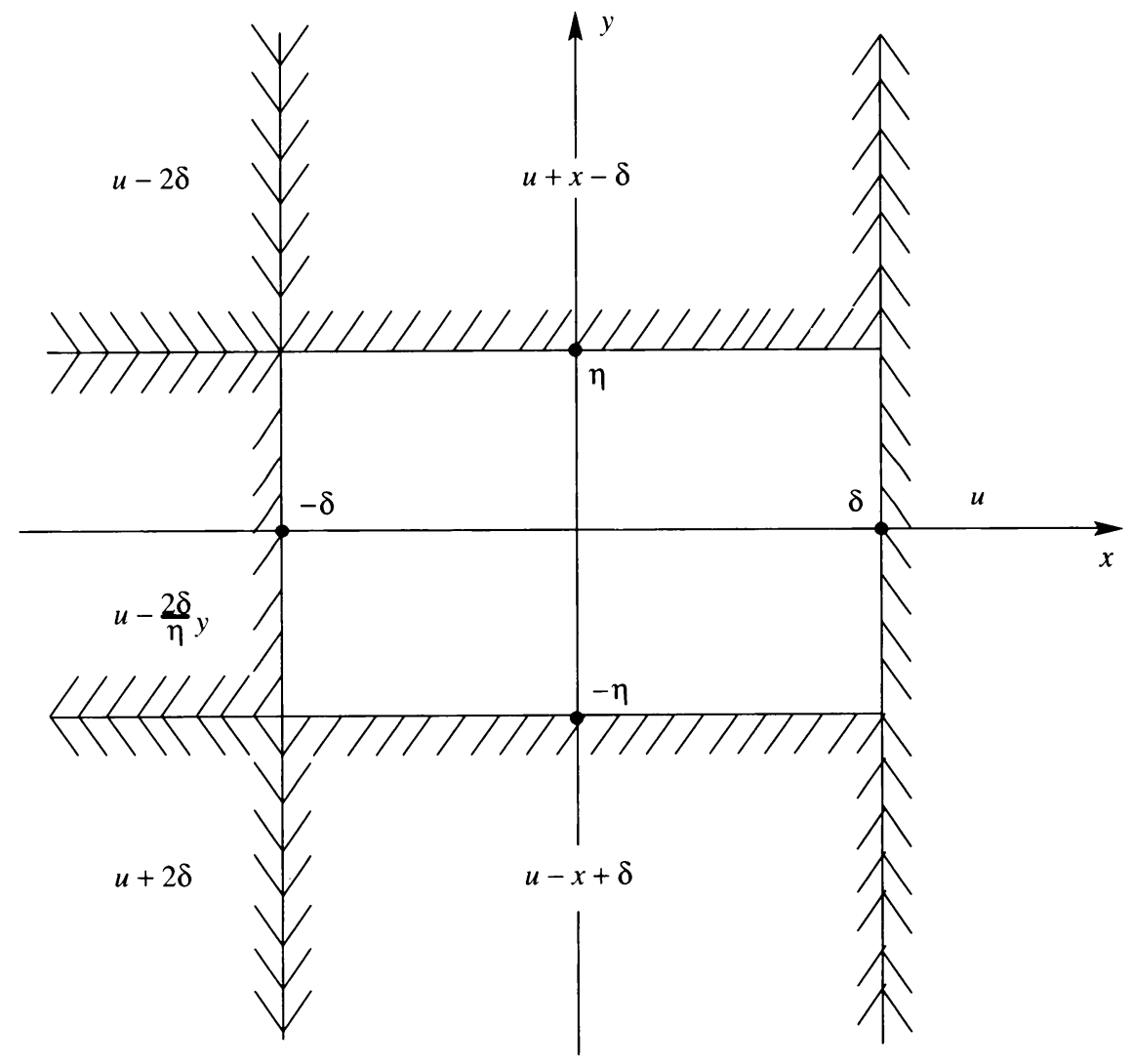

Fig. 4. Illustration of Liapunov function (5). 
Then, we obtain from ( $\Lambda$.2) that $V(x, y) \rightarrow+\infty$ as $|x|+|y| \rightarrow+\infty$. Now, define

$$
\dot{V}(x, y)=\limsup _{h \rightarrow 0^{+}}\{V(x(t+h))-V(x(t))\} / h .
$$

Then, $\dot{V}(x, y)$ can be calculated as follows.

(I) When $(x, y) \in\left\{(x, y) \in \mathbf{R}^{2}: x \geq \delta, y \in \mathbf{R}^{1}\right\} \cup\left\{(x, y) \in \mathbf{R}^{2}: x \leq-\delta,|y| \geq\right.$ $\eta\}$, we have from $(\Lambda .1)$ and $(\Lambda .3 .1)$ that

$$
\dot{V}(x, y)=g(x)\{f(x, y)-f(0, y)\}<0 .
$$

(II) When $(x, y) \in\left\{(x, y) \in \mathbf{R}^{2}:|x| \leq \delta, y \geq \eta(\geq \zeta)\right\}$, we have from (3.1)

$$
\begin{aligned}
\dot{V}(x, y) & =\{f(x, y)-f(0, y)\}\{g(x)+1\}+f(0, y) \\
& \leq\left\{\operatorname{Sup}_{|x| \leq \delta, y \in \mathbf{R}^{\prime}}|f(x, y)-f(0, y)|\right\}\left\{\operatorname{Sup}_{|x| \leq \delta}|g(x)+1|\right\}+f(0, y)<0 .
\end{aligned}
$$

(III) When $(x, y) \in\left\{(x, y) \in \mathbf{R}^{2}:|x| \leq \delta, y \leq-\eta(\leq-\zeta)\right\}$, by an argument similar to that of (II), it follows from (3.2) that $\dot{V}(x, y)<0$.

(IV) When $(x, y) \in\left\{(x, y) \in \mathbf{R}^{2}: x \leq-\delta,|y| \leq \eta\right\}$, we have from (4)

$$
\begin{aligned}
\dot{V}(x, y) & =g(x)\{f(x, y)-f(0, y)-2 \delta / \eta\} \\
& \leq g(x)\{f(x, y)-f(0, y)-2 \delta / \theta\}<0 .
\end{aligned}
$$

From (I)-(IV), we see

$$
(x, y) \in\left\{(x, y) \in \mathbf{R}^{2}:|x| \geq \delta\right\} \cup\left\{(x, y) \in \mathbf{R}^{2}:|y| \geq \eta\right\} \rightarrow \dot{V}(x, y)<0 .
$$

The ultimate boundedness of $\Lambda$ follows directly from (6) and Theorem 14.6 of Yoshizawa [19]. Thus, the Poincaré-Bendixson theorem completes the proof.

As shown later, $(\Lambda .3)$ and $(\Lambda .4)$ also provide a generalization of the well-known Kaldor's condition.

REMARK 1. The Liapunov function analogous to (5) in the proof of Theorem 2 was first ascertained by Mizohata and Yamaguchi [13]. They used it to detect a periodic solution of the system $d^{2} x / d t^{2}+f(x) d x / d t+g(x)=p(t)$, which is equivalent to $d x / d t=-y-\int^{x} f(s) d s+\int^{t} p(\tau) d \tau, d y / d t=-g(x)$. The Liapunov function (5) gives a generalization of Mizohata and Yamaguchi's.

The next corollary with slightly stronger conditions is also useful for guaranteeing the outermost and innermost limit cycles for the business cycle models in the Keynesian tradition.

Corollary 2. Under $(\Lambda .4)$ and the following assumptions, $\Lambda$ has an outermost limit cycle that is externally stable and an innermost limit cycle that is internally stable.

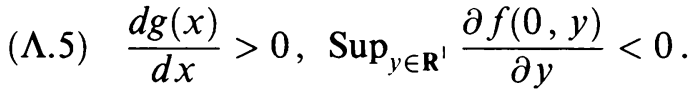

$(\Lambda .6)$ There exists a positive number $\delta$ such that

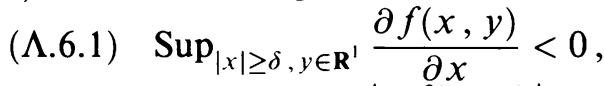

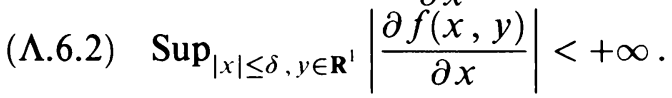


Proof. See the proof of Corollary 1.

4. Two-sided stability. So far, we have considered the existence of outermost and innermost limit cycles that display one-sided stability. It is important, from the business cycle theoretic point of view, to know what sorts of conditions guarantee two-sided stability of an emerging cycle. An important uniqueness-stability theorem by Huang Kecheng (see Ye Yanqian [18, p.156]) gives a sufficient condition for guaranteeing the stability of a cycle in the Liénard system. His result is, with several modifications, as follows.

Theorem 3 (Huang Kecheng). Consider the system $\Lambda^{\sharp}$ :

$$
\begin{aligned}
d x / d t & =-y-F(x), \\
d y / d t & =g(x),
\end{aligned}
$$

where $F$ is of class $C^{1}, g$ is continuous, and $F(0)=0$. Suppose the following conditions are satisfied:

$\left(\Lambda^{\sharp} .1\right) \quad g(x) x>0$ for any $x \neq 0$,

$\left(\Lambda^{\sharp} .2\right)$ there exist numbers $a, a^{\prime}, b$, and $b^{\prime}\left(b^{\prime}<a^{\prime}<0<a<b\right)$ such that

$\left(\Lambda^{\sharp}\right.$.2.1) $\quad F(x) \leq F(a)$ when $0 \leq x \leq a$, and $F(x)$ is monotonically increasing on $[a, b]$,

$\left(\Lambda^{\sharp}\right.$.2.2.) $\quad F(x) \geq F\left(a^{\prime}\right)$ when $a^{\prime} \leq x \leq 0$, and $F(x)$ is monotonically increasing on $\left[b^{\prime}, a^{\prime}\right]$.

Then, in the strip $b^{\prime} \leq x \leq b, \Lambda^{\sharp}$ has at most one limit cycle.

Proof. See Ye Yanqian [18, pp. 156-158].

Directly from Theorem 2 and Theorem 3 , we obtain

THEOREM 4. If $\left(\Lambda^{\sharp} .1\right)$ and the following conditions are all satisfied, then $\Lambda^{\sharp}$ has a unique stable limit cycle.

$\left(\Lambda^{\sharp} .3\right)$ There exists a positive number $\beta$ such that

$$
\operatorname{Inf}_{|y| \geq \beta}|g(x)|>0 .
$$

$\left(\Lambda^{\sharp} .4\right)$ There exists a positive number $\delta$ such that

$$
\operatorname{Inf}_{|x| \geq \delta} \frac{d F(x)}{d x}>0 .
$$

$\left.\left(\Lambda^{\sharp} .5\right) \quad \frac{d F(x)}{d x}\right|_{x=0}<0$.

Proof. From Theorem 2, we see that, under the conditions of Theorem 4, $\Lambda^{\sharp}$ has an outermost limit cycle that is externally stable and an innermost limit cycle that is internally stable.

On the other hand, from $\left(\Lambda^{\sharp} .4\right)$, there exist sufficiently large numbers $a$ and $a^{\prime}\left(a^{\prime}<-\delta<0<\delta<a\right)$ such that

$$
F(a) \geq \operatorname{Sup}_{x \in[0, a]} F(x) \text { and } F\left(a^{\prime}\right) \leq \operatorname{Inf}_{x \in\left[a^{\prime}, 0\right]} F(x) .
$$


We see from $\left(\Lambda^{\sharp} .4\right)$ that, for these numbers and any numbers $b, b^{\prime}$ that satisfy $b>a$ and $b^{\prime}<a^{\prime},\left(\Lambda^{\sharp} .2\right)$ of Theorem 3 is satisfied. Thus, we see from Theorem 3 that $\Lambda^{\sharp}$ has at most one cycle in $\mathbf{R}^{2}$. This implies the coincidence of outermost and innermost limit cycles. Thus, we complete the proof.

5. Existence of persistent business cycles. Results in the previous sections have applications to several business cycle models in the Keynesian tradition. In the following, we show it.

(I) As discussed by Chang and Smyth [3], the most general form of the net-value version of the Kaldor model is given by $\mathrm{KA}$ :

$$
\begin{aligned}
& d Y / d t=\alpha\{I(Y, K)-S(Y)\}, \\
& d K / d t=\lambda I(Y, K)+(1-\lambda) S(Y),
\end{aligned}
$$

where $I$ is the net investment, $S$ savings, $Y$ the net income, $K$ the capital stock, $\alpha$ the adjustment coefficient (positive constant), and $\lambda$ a nonnegative constant $(0 \leq \lambda \leq$ 1). This model was named the "Kaldor model" after its first discoverer, N. Kaldor [9]. But the mathematically exact formalization of the model was first given by Chang and Smyth [3].

For KA with $\lambda \neq 0$, we can show, by means of the geometrical technique of Chang and Smyth [3], the existence of outermost and innermost limit cycles. But, for the same reason as stated in the introduction, their technique does not hold true for the case $\lambda=0$. KA with $\lambda=0$ was first constructed by Ichimura [8]. In this case, the actual change in the capital stock is determined by savings decisions. The planned savings is always realized and equal to the realized investment, and, therefore, equal to $d K / d t$. But the planned investment is not always realized.

We first provide a sufficient condition that guarantees outermost and innermost limit cycles for any $\lambda \in[0,1]$. For KA, as usual, we assume

$$
\frac{\partial I}{\partial Y}>0, \quad \frac{\partial I}{\partial K}<0, \quad 0<\frac{d S}{d Y}<1 .
$$

Note that the equilibria of KA for any $\mu \in[0,1]$ correspond to those of KA with $\lambda=0$. On the other hand, since the line $d K / d t=0$ is vertical in the $(Y, K)$ phase diagram, we see that, under the conditions above, KA has a unique equilibrium point. We can suppose, after a suitable translation, that the unique equilibrium point is the origin. Then, each of $Y$ and $K$ denotes the deviation from the equilibrium.

We observe directly from Corollaries 1 and 2 that

COROLlaRY 3. Under the following conditions, KA for any $\lambda \in[0,1]$ has an outermost limit cycle that is externally stable and an innermost limit cycle that is internally stable.

(KA.1)

$$
\begin{aligned}
& \operatorname{Sup}_{(Y, K) \in \mathbf{R}^{2}} \frac{\partial I}{\partial K}<0, \quad \operatorname{Sup}_{(Y, K) \in \mathbf{R}^{2}}\left|\frac{\partial I}{\partial K}\right|<+\infty, \\
& \operatorname{Sup}_{(Y, K), \in \mathbf{R}^{2}}\left|\frac{\partial I}{\partial Y}\right|<+\infty, \quad \operatorname{Inf}_{Y \in \mathbf{R}^{\prime}} \frac{d S}{d Y}>0 .
\end{aligned}
$$


(KA.2) There exists a positive number $\delta$ such that

$$
\operatorname{Sup}_{Y \geq \delta}\{I(Y, K)-I(0, K)-S(Y)\}<0, \quad \operatorname{Inf}_{Y \leq-\delta}\{I(Y, K)-I(0, K)-S(Y)\}>0 .
$$

(KA.3) Any eigenvalue of the Jacobian matrix of KA that is evaluated at $(0,0)$ has a positive real part.

Specifically, (KA.2) can be replaced by

(KA.4) There exists a positive number $\delta$ such that

$$
\operatorname{Sup}_{|Y| \geq \delta} \frac{\partial(I-S)}{\partial Y}<0
$$

(KA.1) is the economically natural assumption. Here, it should be noted that

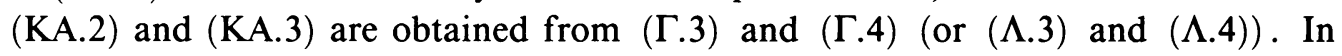
a specified form of the investment function $I(Y, K)=L_{1}(Y)+L_{2}(K)$, Kaldor's condition is stated as $(\mathrm{i})$ there are numbers $Y_{1}$ and $Y_{2}\left(Y_{1}>0>Y_{2}\right)$ such that $\operatorname{Sup}\left\{L_{1}(Y)-L_{1}(0)-S(Y)\right\}>0$ when $Y>Y_{1}$ and $\operatorname{Inf}\left\{L_{1}(Y)-L_{1}(0)-S(Y)\right\}>0$ when $Y<Y_{2}$, and (ii) $d\left(L_{1}-S\right) / d Y>0$ when $Y=0$. See Chang and Smyth [3] and Gabisch and Lorenz [5]. (i) and (ii) are obtained from (KA.2) and (KA.3). Thus, we see that $(\Gamma .3)$ and $(\Gamma .4)$ (or $(\Lambda .3)$ and $(\Lambda .4)$ ) essentially give a mathematical generalization of Kaldor's condition.

Next, we consider stability of the limit cycle under consideration. By introducing strong symmetry hypotheses, such as the property that the investment and savings functions are symmetric with respect to the equilibrium point, Lorenz [11] has proved that the well-known Levinson-Smith theorem [10] can be applied to KA. As suggested by Lorenz, it is, however, very rare that the hypotheses are satisfied. But more recently, Galeotti and Gori [6] showed, by means of the result of Zhang Zhifen [20], that these strong hypotheses can be excluded in the case that $\partial I / \partial K \equiv$ const. We start by stating their result in a slightly more extensive form.

Theorem 5 (Galeotti and Gori). Suppose $I$ and $S$ are of class $C^{2}$. Under the above-mentioned three conditions (i.e., $\partial I / \partial Y>0, \partial I / \partial K<0$, and $1>d S / d Y>$ $0)$ and the following conditions, KA has a unique stable limit cycle:

(i) $\partial I / \partial K \equiv$ const

(ii) $\partial I / \partial Y+(\lambda / \alpha) \cdot \partial I / \partial K>0$,

(iii) $\{I(Y,(\lambda / \alpha) Y)-S(Y)\}$ is concave for $Y>0$ and convex for $Y<0$.

Specifically if (ii) with $\lambda=1$ is satisfied, then $\mathrm{KA}$ for any $\lambda \in[0,1]$ has a unique stable limit cycle.

Proof. Consider the next transformation, which is slightly more general than that utilized by Galeotti and Gori [6]:

$$
(x, y)=(Y, K-(\lambda / \alpha) Y) .
$$


By this transformation, KA becomes $\mathrm{KA}^{\sharp}$ :

$$
\begin{aligned}
& d x / d t=\alpha\{I(x, y+(\lambda / \alpha) x)-S(x)\}, \\
& d y / d t=S(x) .
\end{aligned}
$$

By applying the method of Galeotti and Gori [6] to $\mathrm{KA}^{\sharp}$, we can complete the proof.

REMARK 2. In Corollary 3 , by applying Corollary 2 to the original system KA, we derived a sufficient condition for the existence of a limit cycle of KA. By applying Corollary 2 to $\mathrm{KA}^{\sharp}$, we can also derive a different existence condition. However, it should be noted that such a condition depends on the magnitudes of the adjustment coefficient $\alpha$ and the parameter $\lambda$. The sufficient condition of Corollary 3 is independent of these parameters. In this sense, as for the existence of a limit cycle, the sufficient condition of Corollary 3 is economically more meaningful.

Galeotti and Gori's result surely excludes such economically meaningless symmetry hypotheses as Lorenz [11] suggested. But the hypotheses employed by Galeotti and Gori [6], i.e., the right-hand concavity and the left-hand convexity of $\{I-S\}$ with respect to equilibrium income, are still economically ad hoc. There is a very strong possibility that such hypotheses may be broken down. See Fig. 5. In the following, we exclude these strong hypotheses.

Corollary 4. Suppose (KA.1) of Corollary 3 and (i) of Theorem 5 are satisfied. Then KA has a unique stable limit cycle if

(KA .5) there exists a positive number $\delta$ such that

$$
\operatorname{Inf}_{|Y| \geq \delta}\left\{\frac{d S(Y)}{d Y}-\frac{\partial I(Y, 0)}{\partial Y}-\frac{\lambda}{\alpha} \cdot \frac{\partial I}{\partial K}\right\}>0,
$$

(KA.6)

$$
\left.\left\{\frac{d S(Y)}{d Y}-\frac{\partial I(Y, 0)}{\partial Y}-\frac{\lambda}{\alpha} \cdot \frac{\partial I}{\partial K}\right\}\right|_{Y=0}<0
$$

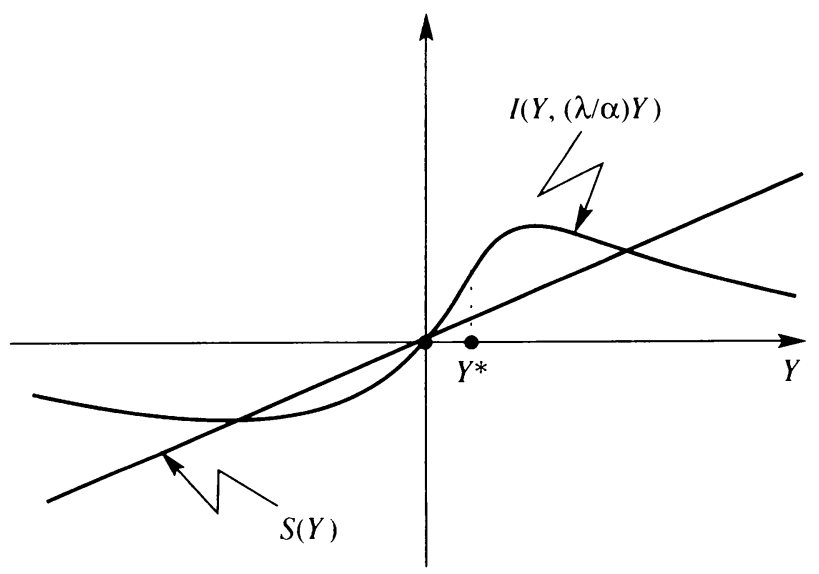

FIG. 5. The case in which Condition (iii) is not satisfied. $Y^{*}$ is a point of inflection. 
Proof. Following Galeotti and Gori [6], we first transform $\mathrm{KA}^{\sharp}$ to a simpler system. Define $P(x, y)=I(x, y+(\lambda / \alpha) x)$ and $Q(x)=S(x)$. Differentiating the first equation of $\mathrm{KA}^{\sharp}$, we have

$$
\frac{d^{2} x}{d t^{2}}=\mu\left(\frac{\partial P}{\partial x}-\frac{\partial Q}{\partial x}\right) \frac{d x}{d t}+\mu \frac{\partial P}{\partial y} Q(x) .
$$

Here, note that, by (i) of Theorem 5 , we have $\partial P / \partial y \equiv$ const $<0$. Consider the transformation

$$
(z, w)=\left(x,-\frac{d x}{d t}+\mu \int_{0}^{x} \frac{\partial\{P-Q\}}{\partial x} d x\right) .
$$

$\mathrm{KA}^{\sharp}$ is transformed to $\mathrm{KA}^{\sharp \sharp}$ :

$$
\begin{aligned}
d z / d t & =-w-\mu\{Q(z)-P(z, 0)\}, \\
d w / d t & =(-\mu \partial P / \partial y) Q(x) .
\end{aligned}
$$

Here, note that $z=x=Y$ and

$$
\begin{aligned}
Q(z) & -P(z, 0)=S(x)-I(x, x / \mu)=S(x)-I(x, 0)-\{I(x, \lambda x / \alpha)-I(x, 0)\} \\
& =S(x)-I(x, 0)-\int_{0}^{(\lambda / \alpha) x} \frac{\partial I}{\partial K} d K=S(x)-I(x, 0)-\frac{\lambda}{\alpha} \frac{\partial I}{\partial K} x .
\end{aligned}
$$

Applying Theorem 4 to $\mathrm{KA}^{\sharp \sharp}$, we complete the proof.

REMARK 3. It should be noted that the two-sided stability condition of Corollary 4, like that obtained by Galeotti and Gori [6], depends on the magnitudes of the adjustment coefficient $\alpha$ and the parameter $\lambda$.

(II) Consider the model of Schinasi [17]: SC:

$$
\begin{aligned}
d Y / d t & =\alpha\{I(Y, H(Y, M))-S(Y-T(Y))+G-T(Y)\}, \\
d M / d t & =G-T(Y),
\end{aligned}
$$

where $Y$ is the net income, $M$ the real money supply, $I$ the net investment, $S$ savings, $H$ the interest rate function, $T$ the tax collections, $G$ the constant government expenditure, and $\alpha$ the adjustment coefficient (positive constant). $H$ is derived as follows. Let $R$ denote the interest rate. $R$ is determined by the equation $L(Y, R)=M$, where $L$ is the function of demand for money. As usual, we assume $\partial L / \partial R>0$ for all $(Y, R)$. Therefore, this equation can be solved with respect to $R$. We denote the solution by the function $H$, i.e., $R=H(Y, M)$ and $L(Y, H(Y, M)) \equiv M$. As usual, we assume

$$
\frac{\partial I}{\partial H}<0, \quad \frac{\partial I}{\partial Y}>0, \quad 0<\frac{d S}{d Y^{t}}<1, \quad 0<\frac{d T}{d Y}<1, \quad \frac{\partial L}{\partial Y}>0, \quad \frac{\partial L}{\partial R}<0 .
$$

Note that under these conditions SC has a unique equilibrium point. We can suppose, after a suitable translation, that the unique equilibrium point is the origin. Then, each of $Y$ and $K$ denotes the deviation from the equilibrium. Note that

$$
\frac{\partial H}{\partial Y}=-\left(\frac{\partial L}{\partial Y}\right) /\left(\frac{\partial L}{\partial R}\right)>0 .
$$

We obtain from Corollary 2 the following. 
Corollary 5. Under the following conditions, SC has an outermost limit cycle that is externally stable and an innermost limit cycle that is internally stable.

(SC.1)

$$
\operatorname{Inf}_{(Y, M) \in \mathbf{R}^{2}} \frac{\partial I}{\partial H} \cdot \frac{\partial H}{\partial M}>0 .
$$

(SC.2) There exists a positive number $\delta$ such that

$$
\begin{gathered}
\operatorname{Sup}_{|Y| \geq \delta, M \in \mathbf{R}^{\prime}}\left\{\frac{\partial I}{\partial H} \cdot \frac{\partial H}{\partial Y}+\frac{\partial I}{\partial Y}-\frac{d T}{d Y}-\frac{d S}{d Y^{t}}\left(1-\frac{d T}{d Y}\right)\right\}<0, \\
\operatorname{Sup}_{|Y| \leq \delta, M \in \mathbf{R}^{\prime}}\left|\frac{\partial I}{\partial H} \cdot \frac{\partial H}{\partial Y}+\frac{\partial I}{\partial Y}\right|<+\infty .
\end{gathered}
$$

(SC.3)

$$
\left.\left\{\frac{\partial I}{\partial H} \cdot \frac{\partial H}{\partial Y}+\frac{\partial I}{\partial Y}-\frac{d T}{d Y}-\frac{d S}{d Y^{t}}\left(1-\frac{d T}{d Y}\right)\right\}\right|_{(0,0)}>0 .
$$

Proof. By the transformation $M=-M^{\sharp}, \mathrm{SC}$ is translated into $\mathrm{SC}^{\sharp}$ :

$$
\begin{aligned}
d Y / d t & =\alpha\left\{I\left(Y, H\left(Y,-M^{\sharp}\right)\right)-S(Y-T(Y))+G-T(Y)\right\}, \\
d M^{\sharp} / d t & =T(Y)-G .
\end{aligned}
$$

By applying Corollary 2 to $\mathrm{SC}^{\sharp}$, we can easily prove the corollary.

(SC.1) is the economically natural assumption. Moreover, (SC.2) and (SC.3) are essentially equivalent to Kaldor's condition.

Schinasi [17] assumed the existence of a compact set on the boundary of which the vector field of SC points inward. Under this assumption, he discussed the existence of at least one limit cycle. Whether or not such a compact set does exist has remained unproved so far. Therefore, his existence proof is unsatisfactory. But we showed, by means of Liapunov's second method, that the Kaldor-type condition guarantees existence of at least one limit cycle.

Next, we consider stability of the limit cycle under consideration. Suppose the specified functional forms:

$$
\begin{aligned}
I(Y, r) & =I_{1}(Y)-a r & & (a>0), \\
T(Y) & =b Y+e & & (0<b<1, e>0), \\
L(Y, r) & =c Y-d r & & (c, d>0) .
\end{aligned}
$$

Here, it should be noted that, since the equilibrium point is the origin, we have $e=T(0)=G$. Then, by the transformation

$$
(Y, M)=(y / b,-(d / \alpha a b) m),
$$

$\mathrm{SC}$ becomes the Lienard system $\mathrm{SC}^{\sharp \sharp}$ :

$$
\begin{aligned}
d y / d t & =-m-\alpha b\left[S(\{(1-b) / b\} y-G)-I_{1}(y / b)+(a c / b d) y+y\right], \\
d m / d t & =(\alpha a b / d) y .
\end{aligned}
$$


Applying Theorem 4 and Corollary 5 to $\mathrm{SC}^{\sharp \sharp}$, we can now easily check that

Corollary 6. Suppose the conditions of Corollary 5 are all satisfied, along with the following conditions:

(SC .4) There exists a positive number $\delta$ such that

$$
\operatorname{Inf}_{|Y| \geq \delta}\left\{\frac{d S((1-b) Y-G)}{d Y}-\frac{d I_{1}(Y)}{d Y}\right\}>0,
$$

(SC.5)

$$
\left.\left\{\frac{d S((1-b) Y-G)}{d Y}-\frac{d I_{1}(Y)}{d Y}\right\}\right|_{Y=0}<0 .
$$

Then SC with the above specified functional forms has a unique stable cycle.

(III) It is also possible to discuss, by the same arguments as in (I) and (II), applications of our results to the Goodwin model [7] and the Benassy model [1]. But we omit the discussions.

Acknowledgments. I would like to thank Tatsuji Owase, Toshio Inaba, and Kazuyuki Sasakura for helpful discussions, and an anonymous referee of this journal for helpful comments and suggestions which greatly improved the exposition of this paper. All errors that may exist are, of course, solely my responsibility.

\section{REFERENCES}

[1] J. P. Benassy, A non-Walrasian model of the business cycle, J. Econom. Behavior Org. 5, 77-89 (1984)

[2] L. Cesari, Asymptotic Behavior and Stability Problems in Ordinary Differential Equations, Ergebnisse der Mathematik und ihrer Grenzgebiete, 2nd ed., Springer-Verlag, Berlin-GottingenHeidelberg, 1959

[3] W. W. Chang and D. J. Smyth, The existence and persistence of cycles in a non-linear model: Kaldor's 1940 model re-examined, Rev. Econom. Stud. 38, 37-44 (1971)

[4] A. F. Filippov, A sufficient condition for the existence of a stable limit cycle for a second order equation, Mat. Sb. 30, 171-180 (1952) (Russian)

[5] G. Gabisch and H.-W. Lorenz, Business Cycle Theory, Lecture Notes in Econom. Math. Systems, Vol. 283, Springer-Verlag, Berlin, 1987

[6] M. Galeotti and F. Gori, Uniqueness of periodic orbits in Liénard-type business-cycle models, Metroeconomica 40, 135-146 (1989)

[7] R. M. Goodwin, The nonlinear accelerator and the persistence of business cycles, Econometrica 19, $1-17(1951)$

[8] S. Ichimura, Towards a general non-linear macrodynamic theory of economic fluctuations, PostKeynesian Economics (K. K. Kurihara, ed.), Rutgers University Press, New Brunswick, 1955

[9] N. Kaldor, A model of the trade cycle, Econom. J. 50, 78-92 (1940)

[10] N. Levinson and O. K. Smith, A general equation for relaxation oscillations, Duke Math. J. 9, 382-403 (1942)

[11] H. W. Lorenz, On the uniqueness of limit cycles in business cycle theory, Metroeconomica 38, 281-293 (1987)

[12] H. W. Lorenz, Nonlinear Dynamical Economics and Chaotic Motion, Lecture Notes in Econom. Math. Systems, Vol. 334, Springer-Verlag, Berlin, 1989

[13] S. Mizohata and M. Yamaguchi, On the existence of periodic solutions of the nonlinear differential equation $\ddot{x}+a(x) \dot{x}+\Phi(x)=p(t)$, Mem. Coll. Sci. Univ. Kyoto, Ser. A 27, 109-113 (1952)

[14] T. Owase, Dynamical system theory and analysis of economic fluctuations, The Study of Dynamical Systems (N. Aoki, ed.), World Scientific, Singapore, 1989

[15] T. Owase, Nonlinear dynamical systems and economic fluctuations: A brief historical survey, Trans. IEICE. E74, 1393-1400 (1991) 
[16] G. Sansone and R. Conti, Non-linear Differential Equations, Macmillan, New York, 1964

[17] G. J. Schinasi, Fluctuations in a dynamic, intermediate-run IS-LM model: Applications of the Poincaré-Bendixon theorem, J. Econom. Theory 28, 369-375 (1982)

[18] Ye Yanqian, Theory of Limit Cycles, Translations of Mathematical Monographs, Vol. 66, Amer. Math. Soc., Providence, R.I., 1986

[19] T. Yoshizawa, Stability Theory by Liapunov's Second Method, Mathematical Society of Japan, Tokyo, 1966

[20] Zhang Zhifen, On the uniqueness of the limit cycles of some nonlinear oscillation equations, Dokl. Akad. Nauk. SSSR 119, 659-662 (1958) (Russian) 